\title{
A Survey on Barcode Generation and Modulation Techniques
}

\author{
Vidyashree Kokane ${ }^{1}$, Shyam Gupta ${ }^{2}$ \\ ${ }^{1}$ PG Student, Siddhant College of Engineering, Sudumbre, Savitribai Phule Pune University \\ ${ }^{2}$ Professor, Computer Department, Siddhant College of Engineering, Sudumbre, Savitribai Phule Pune University
}

\begin{abstract}
Between handheld electronic devices in wireless data transmission for use the concept of HC2D barcodes is of great relevance. In a typical setup, through a images series on the LCD there can be transferred any file on a cell phone to a second cell phone through the camera of the second cell phone which are then captured and decoded. In this study, in HC2D barcodes a new approach for data modulation is introduced, and to other standard methods of barcode modulation its performance is evaluated in comparison. In this new approach, within neighboring pixels of an LCD over adjacent blur, and light leakage, there is used the orthogonal frequency-division multiplexing (OFDM) modulation together with differential phase shift keying (DPSK).
\end{abstract}

Keywords: HC2D barcode, data transfer, differential phase shift keying, orthogonal frequency-division multiplexing (OFDM) modulation.

\section{Introduction}

In facilitating numerous identification processes Barcodes have played a great role since their invention in 1952 [1]. In fact for machine readable digital data storing on product packages or paper, barcode is a cost-effective and simple method. Even faster data transfer as pressing needs and there have been many improvements with high reliability have emerged on the original barcode design that was made. For these cost-effective codes as well as their application opened a new front by invention of HC2D barcodes in scenarios like storing contact information which transfer more complex data, URLs among other things, there have become increasingly popular in which QR codes [2]. There can be found the performance of camera phone applications in the comparison of 2D barcode in [3]. In matrix barcode development there have been dedicated much of the efforts on a piece of paper to barcodes displayed they are normally used as that is the way. That replacement of the paper with LCD One could contemplate with tablets and e-Book readers replacement with the books for broader applications of $2 \mathrm{D}$ barcodes may open another promising front as a mean of data transfer. Moreover for the eventual streams of data transfer, the LCD may display time-varying barcodes unlike the static paper to the receiving electronic device(s).

Through a series of HC2D barcode there transfer the data between two cell phones is studied, for state of the art mobile devices which achieving bit rates of under $10 \mathrm{kbps}$. With bit rates of more than $14 \mathrm{Mbps}$ later for transmission and reception, a digital camera and a computer monitor are used. Over distances of up to 4 meters which achieved in docked transmitter and receiver conditions. However, when the distance is increased to 14 meters this rate drops to just over 2 Mbps. Using a more effective coding and modulation scheme there is achieved the superior performance of the later implementation for mitigation of pixel and image blur to pixel light leakage. To modulate LCD pixels there used the inverse Fourier transform (IFT) of data like OFDM. The performance of QR decoders reduces by image light and blurs leakage greatly. Furthermore to known portions of the decoded data there is confined their performance degradation. Based on data region as in [5] on non-uniform error probability for adaptive error correction may be used coding this prior knowledge. In design as well as implementation of LCD-Camera based communication systems there is an increasing interest as indicated in [6]-[8]. For this type of innovative communications medium in determining optimal demodulation and modulation schemes this would require additional investigations.

For transferring data, there uses orthogonal frequency subcarriers by the OFDM modulation and image blur can confine, to high frequency components which is essentially a low pass filter are transmitted intact. To detect the data bits correctly this method requires high phase coherency. Through additional modifications this idea extends by the current study during the capture of a single frame in a way on the modulation scheme for mitigating LCD-camera relative movements, on the captured images which results in distortion of motion blur. As would be detailed later severely, the Quadrature Phase Shift Keying (QPSK) performance modulated signals of OFDM degrade due to this kind of distortion. From a single image which is shown on an LCD one should consider extracting maximum data to maximize data transmission rate and then at which consecutive frames will be the rate decoded increase.

\section{Literature Survey}

A. QR Code Detection in Arbitrarily Acquired Images Through creation of links between Internet resources and physical objects, there enable rich context interaction by 


\section{International Journal of Science and Research (IJSR) \\ ISSN (Online): 2319-7064}

Index Copernicus Value (2013): 6.14 | Impact Factor (2013): 4.438

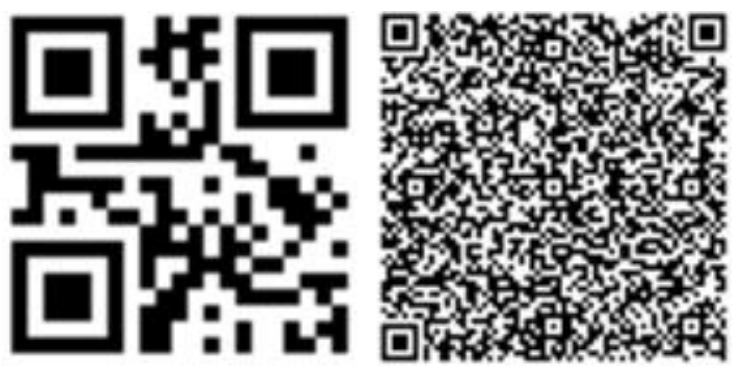

Figure 1: Different versions of $Q R$ code

applications of Quick Response (QR) codes. There are this kind of barcode applications are not common in spite of the widespread use for people and robots which are visually impaired because during image acquisition that the symbol is properly by existing decoders framed are assumed. To perform accurate detection of QR code symbols a two-stage component-based approach proposed by this work in arbitrarily acquired images.

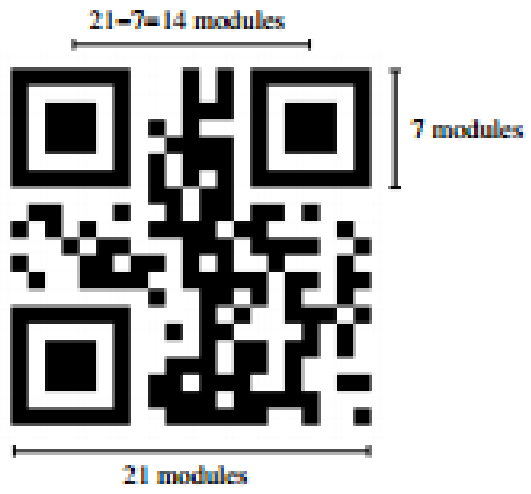

Figure 2: 2D QR code

With the help of the framework which detect the objects proposed by Viola-Jones to detect parts of the symbol is trained, a cascade classifier in the first stage. In the second stage, in order to evaluate the detected patterns are aggregated if they are spatially arranged with the components of a QR code symbol in a way that is geometrically consistent. There was performed an extensive study of both stages parameter variation and in terms of computational efficiency, recall and precision the results were analyzed. With precision of 76: 8\% there achieved average recall of $91: 7 \%$ by the proposed QR code detector while at $22 \mathrm{fps}$ being capable of processing a 640 by 480 pixels video stream. For real-time applications implementation these results support which in mobile hardware assist visually impaired people and robots, through QR codes in multiple medium to the wealth of information available allowing them to have access.

\section{B. 1D bar code reading on camera phones}

In this paper, using a NOKIA 7650, VGA camera phone on the bar code reading algorithms we present the research effort. From poor-quality images to extract bar code characters knowledge-based bar code segmentation and a wavelet-based bar code region location scheme is applied. For the recognition engine there are input all the characters which are segmented bar code, and the bar code character string as the final recognition result with the smallest total distance is output of the bar code based on the recognition distance. For optimizing the class reference vectors and a feature extraction matrix in order to train an efficient recognition

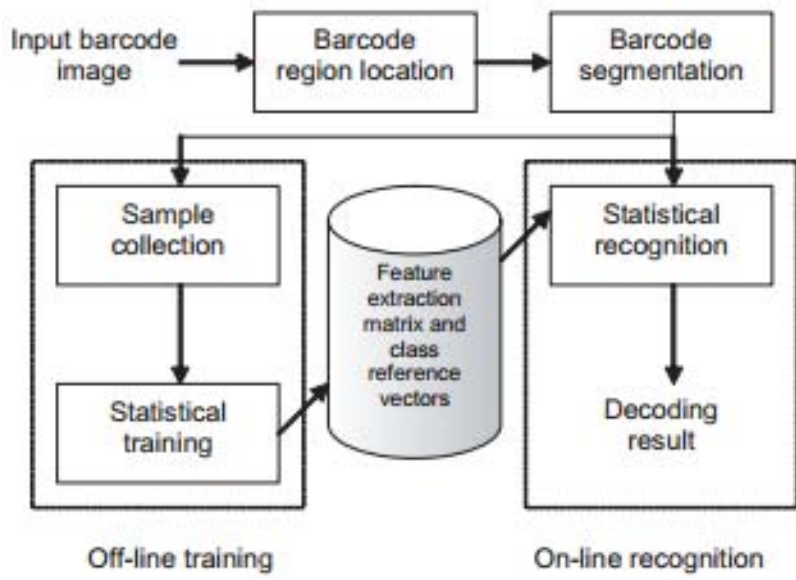

Figure 3: Diagram of the bar code decoding system.

engine, there is designed the modified Generalized Learning Vector Quantization (GLVQ) method. By NOKIA 7650 from more than 1000 bar code images captured, 584 samples segmented are involved in the training process. By the same phone the testing on 292 bar code images taken, there reaches $85.62 \%$ the entire bar code set of the correct recognition rate.

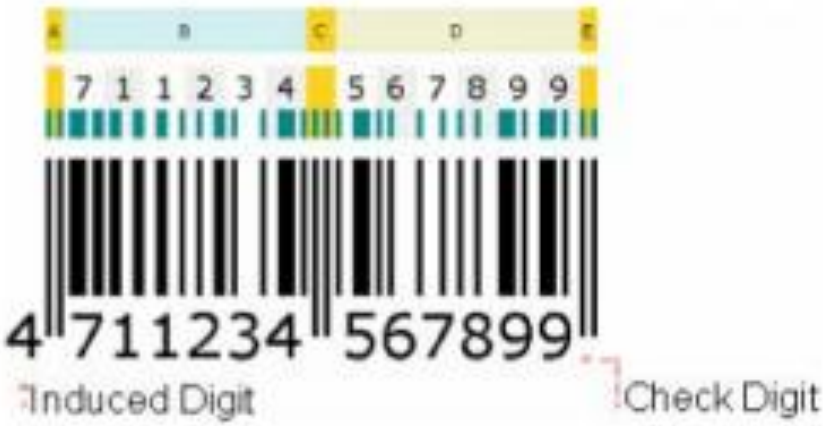

Figure 4: 1D barcode

C. Detecting and Decoding Algorithm for 2D Barcode

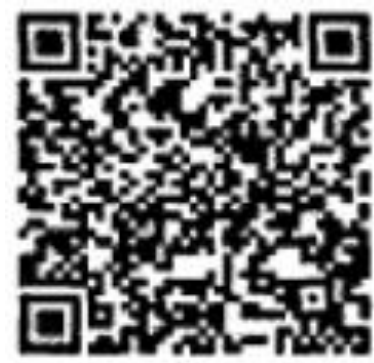

Figure 5: 2D barcode

There can be classified the 2D Barcode mainly in two types which are matrix 2D barcode stacked 2Dbarcode. The structure of types of 2D barcode is discuss in this paper in brief. The flowchart of detecting 2D barcode propose this paper and the 2D barcode also decoding.

\section{Volume 4 Issue 11, November 2015}




\section{International Journal of Science and Research (IJSR) \\ ISSN (Online): 2319-7064}

Index Copernicus Value (2013): 6.14 | Impact Factor (2013): 4.438

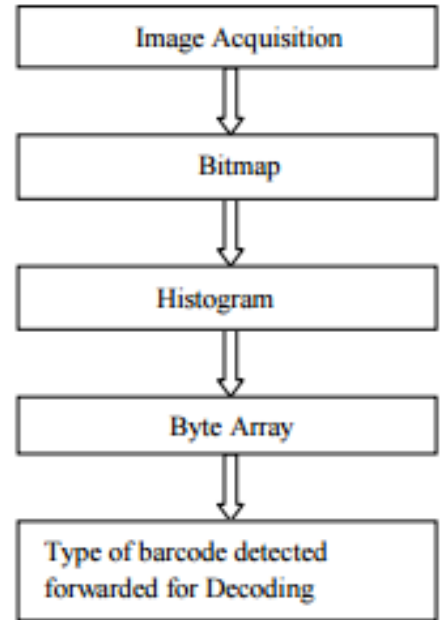

Figure 6: Detecting 2D barcode

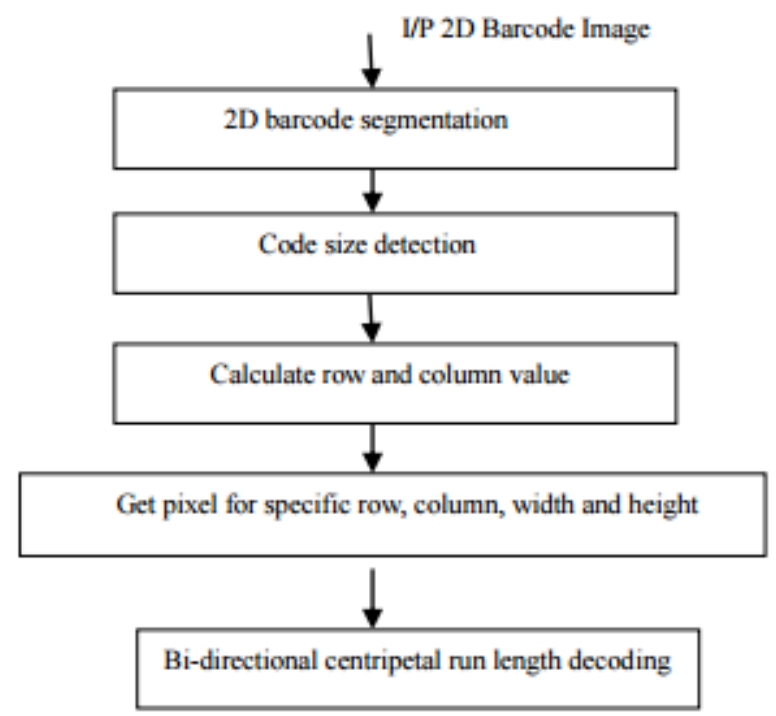

Figure 7: Decoding 2D barcode

D. Linear barcode scanning system based on dynamic template Matching for oof blurred images

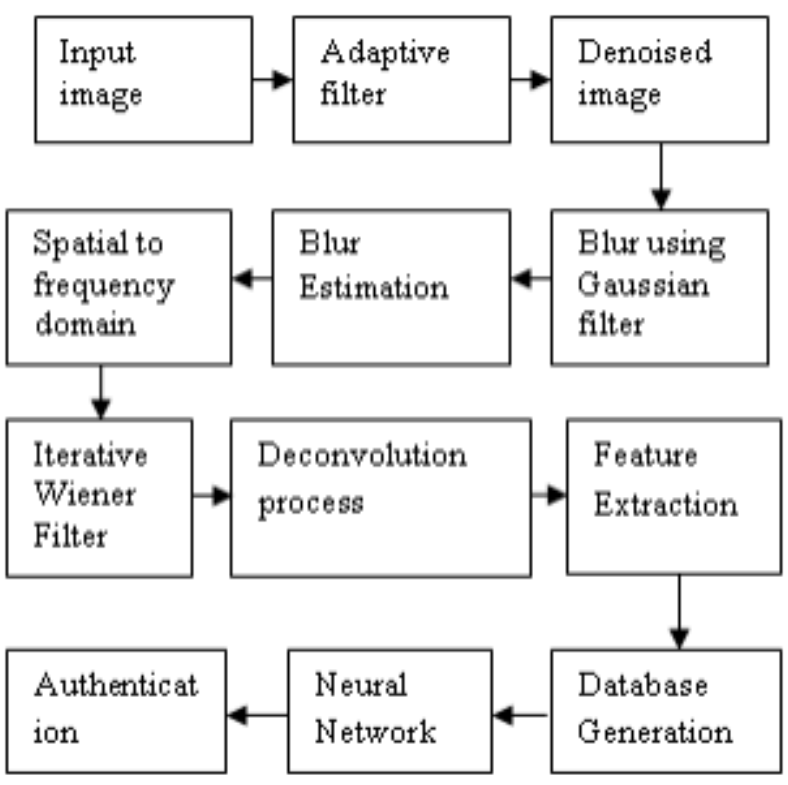

Figure 8: Dynamic template matching
In the spatial domain the proposed system works entirely and from severe OOF blur contain the low-resolution images is capable of reading Linear Barcodes. Under the perspective of deformed Binary waveform classification analysis, this paper treats linear barcode scanning. At any specific blur level for characterizing the relationship between the waveform of blurred barcode and value of its corresponding symbol a directed graphical model is designed. For enabling real-time decoding, retrieve the optimal state sequence there is designed a Dynamic programming-based inference algorithm on mobile devices of limited processing power.

\section{E. Two-Dimensional Barcodes for Mobile Phones}

For a high data density barcode there are several potential applications by mobile phones, that can be easily decoded and photographed, but currently no such symbology exists. As a result, for exploiting the camera phone channel's lowpass characteristic a new barcode was designed and with mobile phones as a means of facilitating wireless optical communication is presented. With encoding done in the Discrete Cosine Transform domain a channel model was established and subsequent simulation results led to the design of a colour barcode.
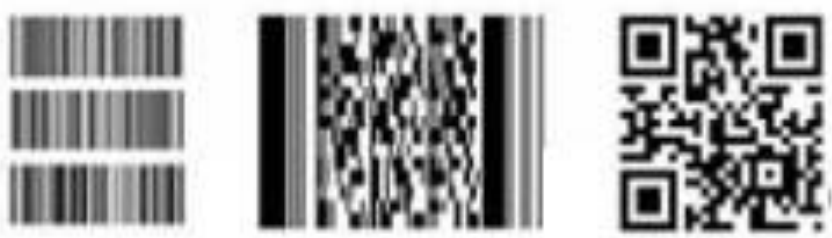

Figure 9: 2D barcodes

There enhance performance by a water-filling process and while for rotational and size invariance a noise-shaping algorithm, a new fast acquisition method allows. According to spatial frequency with a rate varying an outer AccumulateRepeat-Accumulate code is employed, by an inner Reed Muller code which followed.

To various impediments imposed by camera phones for the leading hasproven and symbology robust there is 3.5 times greater the final barcode data-density.

\section{F. QR Inception: Barcode-in-Barcode Attacks}

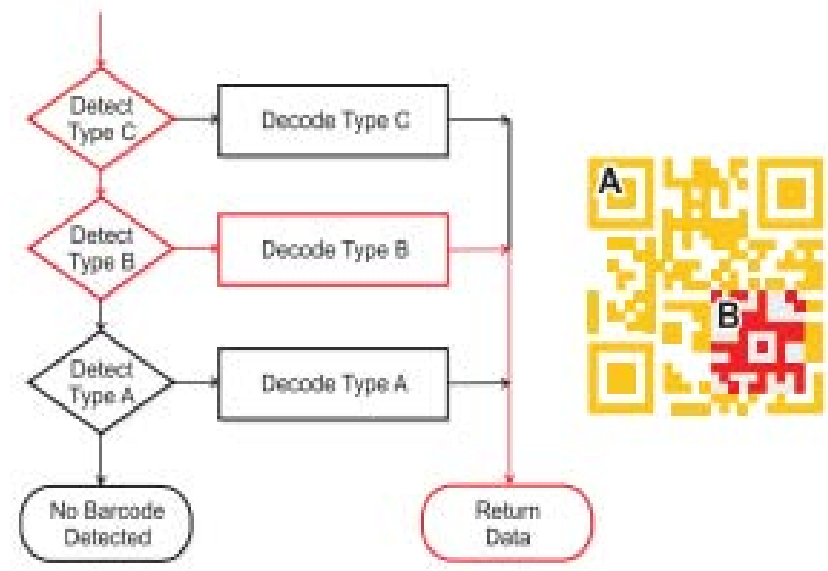

Figure 10: Attack: The inner barcode type is always detected before the outer one 


\section{International Journal of Science and Research (IJSR) \\ ISSN (Online): 2319-7064}

Index Copernicus Value (2013): 6.14 | Impact Factor (2013): 4.438

In this paper, when especially crafted barcodes conform based on deliberately caused ambiguities we present novel attacks to multiple standards. Which standard the decoder locks on decides by implementation details. This way, with different phones or apps two users scanning the same barcode which will receive different content. For multiple problems related to security this potentially opens way. For performing a phishing attacks as well as targeted exploits How embedding one barcode symbology into another is described In addition, for these barcode-in-barcode attacks on smartphones are susceptible popular 2D barcode reader applications is evaluate. Against this type of attack we discuss the mitigation techniques further.

\section{Conclusion}

In this paper in order to data stream modulate into visual HC2D barcodes, there is combined the Differential Phase Shift Keying with Orthogonal Frequency Division Multiplexing. In the mitigation of camera LCD movements there has serious shortcomings that QPSK-OFDM modulation is it shown where there continuously changes the each element phase. On the other hand, from the ideal phase before OFDM a differential phase modulator addition in the received signal because of its change in gradual from element to element, to a small deviation contributing, into phase differences of adjacent elements (DPSK-OFDM) to modulate the data stream causes the effect of motion to increasingly weaken.

\section{References}

[1] N. J. Woodland and B. Silver, Elassifying apparatus and method," U.S. Patent 2612 994, Oct. 7, 1952.

[2] Information Technology-Automatic Identification and Data Capture Techniques-QR Code 2005 Bar Code Symbology Specification, ISO/IEC 18004:2006, 2006.

[3] H. Kato and K. Tan, Pervasive 2d barcodes for camera phone applications," Pervasive Comput., vol. 6, no. 4, pp. $76-85$, Oct. 2007.

[4] X.Liu,D.Doermann,andH.Li, $\vee$ code-pervasive data transfer using video barcode," IEEE Trans. Multimedia, vol. 10, no. 3, pp. 361-371, Apr. 2008.

[5] S. D. Perli, N. Ahmed, and D. Katabi, Pixnet: In terference-free wireless links using LCD-camera pairs," in Proc. MobiCom, 2010, pp. 137-148.

[6] J. Memeti, F. Santos, M. Waldburger, and B. Stiller, Đata transfer using a camera and a three dimensional code," Praxis der Informa tionsverarbeitung und Kommunikation, vol. 36, no. 1, pp. 31-37, 2013.

[7] C. Pei, Z. Zhang, and S. Zhang, Softoc: Real-time projector-wall-camera communication system," in Proc. ICCE, Jan. 2013, pp. 100-101.

[8] S. Kuzdeba, A. M. Wyglinski, and B. Hombs, Prototype implementation of a visual communication system employing video imagery," in Proc. CCNC, 2013, pp. 184-189.

[9] M. Mondal and J. Armstrong, Impact of linear misalignment on a spatial OFDM based pixelated system," in Proc. 18th Asia-Pacific Conf. Commun., Oct. 2012, pp. 617-622.
[10]M. Mondal and J. Armstrong, The effect of defocus blur on a spatial OFDM optical wireless communication system," in Proc. 14th Int. Conf. Transparent Opt. Netw., Jul. 2012, pp. 1-4.

[11] M. R. H. Mondal and J. Armstrong, Analysis of the effect of vignetting on mimo optical wireless systems using spatial OFDM,” J. Lightw. Technol., vol. 32, no. 5, pp. 922-929, Mar. 1, 2014.

[12]R. Gonzalez and R. Woods, Digital Image Processing, 3rd ed. Upper Saddle River, NJ, USA: Pearson Education, 2007.

[13]A. Sripad and D. Snyder, A necessary and sufficient condition for quantization errors to be uniform and white," IEEE Trans. Acoust., Speech, Signal Process. , vol. ASSP-25, no. 5, pp. 442-448, Oct. 1977.

[14] J. Proakis and M. Salehi, Digital Communications, 5th ed. New York, NY, USA: McGraw-Hill Education, 2007.

[15] T. Cover and J. Thomas, Elements of Information Theory,2ndEd. ed. Hoboken, NJ, USA: Wiley, 2006.121-130. 